\title{
Hemodialysis Patient Care in Renal Dialysis Unit among Selected Hospitals in the Sultanate of Oman
}

\author{
Jeffrey Navarro Rojas ${ }^{1}$ \\ Sur Nursing Institute, Ministry of Health, Sultanate of Oman
}

\begin{abstract}
Chronic kidney disease is a major health problem due to the significant financial burden for the healthcare system and likewise for the patient who needs the treatment. The patient's whole life situation is turned upside down with chronic kidney disease when they are confronted with the forced change to start treatment with hemodialysis. Patients undergoing hemodialysis require direct and continuous care. Identifying the barriers to and factors facilitating hemodialysis care can improve care quality. The increasing number of patients with end stage renal disease (ESRD) has caused a substantial rise in the number of individuals receiving hemodialysis. A literature review was conducted through sources of data base available in the Medline/Pub Med, Google Scholar, Science Direct, and EBSCO Academic Search Complete for studies published in Gulf countries. Fourteen studies were found to be related to the domains of hemodialysis patient care in the Sultanate of Oman. Almost 34 percent of the published studies (four studies) were from Saudi Arabia, another four each from Iran, two each form Iraq and Oman respectively, one each from Qatar and the entire GCC. Although the current status of the hemodialysis patient care in the Sultanate of Oman is affected by several factors and necessitates the need for identifying the satisfaction on patient care in renal dialysis unit, concerns of patient undergoing hemodialysis, and health care providers' experiences on patient care in renal dialysis unit, and the barriers and facilitators on patient care in renal dialysis unit. Future studies should be conducted to identify other factors that could influence the hemodialysis patient care in the Sultanate of Oman and the development of interventions to help both the HD patients and the health providers.
\end{abstract}

Keywords: Hemodialysis, Satisfaction, Barriers, Facilitators, Chronic kidney disease

\section{Introduction}

Chronic kidney disease is a major health problem due to the significant financial burden for the healthcare system and likewise for the patient who needs the treatment. The patient's whole life situation is turned upside down with chronic kidney disease when they are confronted with the forced change to start treatment with hemodialysis.

Patients undergoing hemodialysis require direct and continuous care. Identifying the barriers to and factors facilitating hemodialysis care can improve care quality. The increasing number of patients with end stage renal disease (ESRD) has caused a substantial rise in the number of individuals receiving hemodialysis. Chronic kidney disease (CKD) is presently considered a public health issue worldwide and it includes a range of different pathological processes that cause the irreversible loss of kidney function [1]. The survival of the patients depends on renal replacement therapy such as dialysis and transplantation [2]. Among these treatment methods, hemodialysis is a common and successful one to control renal diseases [3].

The number of persons receiving renal replacement therapy (RRT) worldwide is estimated at more than 2.5 million, with the incidence growing by approximately $8.0 \%$ annually [4-5]. In the USA, $91.9 \%$ of renal patients underwent hemodialysis treatment in 2009 [6]. Moreover, hemodialysis (HD) remains the common mode of renal replacement therapy where blood is filtered through an external dialyser performing functions similar to the kidney [7].

Furthermore, HD has a major influence on the everyday life of patients since it involves adjustment to an extensive and complicated treatment regimen where patients are generally required to be present at the hospital three times a week for treatment sessions that last four hours, in combination with continual nutritional management related to dietary and fluid intake, multiple medications as well as recommended exercise.

The provision of care for these patients, before and after the initiation of dialysis, is less than at the most favorable level, resulting in a high financial burden and unfavorable clinical outcomes for patients as well as the health care system [8].

The Gulf Cooperation Council countries (GCC) such as Saudi Arabia, UAE, Kuwait, Qatar, Bahrain and Oman share similar culture and ethnic backgrounds as well as have similar socio-demographic and socioeconomic development.The aim of the present study is to review the published literature on hemodialysis patient care in the Gulf countries. 


\section{Methods}

A literature review was conducted through online sources of data base available in the Medline or PubMed, Google Scholar, Science Direct, and EBSCO Academic Search Complete for published studies in Gulf Countries and nearby areas. Search terms used singly or in combination included: patient satisfaction, hemodialysis, barriers and facilitators, concerns, health care providers, experiences, patient care, renal dialysis unit. Criteria for inclusion were 1) the study is done among any of the Gulf countries such as Kuwait, Bahrain, Oman, Qatar, Saudi Arabia, United Arab Emirates, Iran, Iraq and Egypt; 2) related to any of the satisfaction, concerns, experiences, barriers or facilitator components or domains. No specific range for year of publication was specified, so as to retrieve maximum number of studies. Fifteen studies were found to be related to the topic were able to be retrieved from the web search. But one study was found to be repeating the information. Thus, there were fourteen studies finally considered for the literature review and are summarized under the following subheadings.

\subsection{Status of Hemodialysis in the Gulf Countries}

\section{Results}

A systematic review on the epidemiology of end-stage renal disease in the countries of the Gulf Cooperation Council (GCC) which consist of Saudi Arabia, the United Arab Emirates, Kuwait, Qatar, Bahrain, and Oman using mixed methods among patients on regular dialysis or End Stage Renal Disease (ESRD) for a minimum of at least three months [9]. However, since many outcomes were reviewed, studies that estimated the incidence and prevalence of ESRD as outcomes should not have defined the study population as ESRD population or patients on regular dialysis including those that mainly comprised children or pregnant woman were excluded. Results revealed that forty-four studies included in the review show that the incidence of ESRD has increased while the prevalence and mortality rate of ESRD in the GCC has not been reported sufficiently. The leading primary causes of ESRD recorded in the countries of the GCC is diabetes with the most prevalent co-morbid conditions being Hypertension and Hepatitis C Virus infection; the most common cause of death was cardiovascular disease and sepsis. Therefore, this study highlights that the lack of national renal registries data is a critical issue in the countries of the GCC.

Another study was carried out which focused on the renal placement therapy in the Sultanate of Oman where it was found out that health services have improved accordingly, including services for patients with chronic renal failure [10]. The incidence of end-stage renal failure in this country is estimated to be around 120 million inhabitants annually. The causes of renal failure are still mostly uncertain but assumed to be chronic glomerulonephritis, since many cases at present are advanced stage. However, diabetes mellitus forms $14.5 \%$ of the causes of renal failure in this population. The services for renal failure patients are fully paid for by the government. Furthermore, dialysis services started in Oman in the form of intermittent peritoneal dialysis (IPD) in AI-Nahdha hospital in the capital Muscat in 1980. In the same hospital, hemodialysis was introduced later in 1983. In 1999, there are 345 hemodialysis and three CAPD patients in Oman, while there is none on 1PD. Hemodialysis patients in Oman receive an average of eight hours dialysis per patient weekly and the most common type of permanent vascular access used is arteriovenous fistula. Cuprophane dialyzers are the most commonly used in this country. Furthermore, reuse is not practiced. However, there are only $18.5 \%$ of hemodialysis patients on bicarbonate dialysis. There are currently $23 \%$ of hemodialysis patients on erythropoietin as a replacement therapy for anemia associated with renal failure. Interestingly, only $17.6 \%$ of the hemodialysis patients in Oman have positive antihepatitis $\mathrm{C}$ antibodies. This is rather low in comparison with the nearby countries.

The demographics and outcome of hemodialysis patients during a five years study period in Qatar where the data of all the patients on regular hemodialysis from January $1^{\text {st }} 2002$ to December 31st, 2006 were included in this study was collected from the medical records and entered into an especially designed questionnaire [11]. The prevalence of end stage kidney disease in Qatar is 624 patients per million populations with an incidence of 202 patients per million populations per year. Currently, 278 patients are on hemodialysis, $65 \%$ of them are Qatari; males represent $51 \%$, whereas $44.6 \%$ are between $65-74$ years of age. Diabetic nephropathy is the commonest cause of end stage kidney disease (48\%), followed by primary glomerulonephritis and hypertensive glomerulopathy. Arteriovenous fistula was the vascular access in 57\% of patients. The incidence of Hepatitis B, C and Human immunodeficiency virus had been stable throughout the study period though our hemodialysis population had increased by 1.5 fold. The first and five years survival rates of our patients were 84 and 53\% respectively. Qatar has one of the highest rates of dialysis patients with a good long-term survival report. Peritoneal dialysis remained to be the key solution for the rapidly expanding patients' pool. Maintenance of national registry of dialysis patients and improving our organ transplant program is an essential goal.

The epidemiological transition of end-stage kidney disease in Oman was examined by using the End Stage Kidney Disease (ESKD) data from1983 to 2013 [12]. Data from 1998 to 2013 were obtained through an 
Information Management System. Data before 2008 were collected from patients' files. A questionnaire based on USRDS form 2728 was completed by nephrologists once a citizen reached ESKD. Results show that a total of 4066 forms were completed, with a response rate of $90 \%$ (52\% male). The mean (SD) age was 50.1 (14.0) years. By 31 December 2013, there were 2386 patients alive on RRT, of whom 1206 were on hemodialysis $(50.5 \%), 1080$ were living with a functioning kidney transplant $(45.3 \%)$, and 100 were receiving peritoneal dialysis (4.2\%). The incidence of ESKD on RRT was 21, 75, and 120 per million population in 1983, 2001, and 2013, respectively. Similarly, the prevalence of ESKD was 49, 916, and 2386 in 1983, 2001, and 2013 respectively. Among patients with ESKD on RRT, a progressive rise was seen in diabetic nephropathy, with $5.8 \%, 32.1 \%$, and $46 \%$ in 1983, 2001, and 2013 respectively. This further reveals that the incidence and prevalence of ESKD has increased progressively over last 30 years and this is expected to continue to increase at an even higher rate in view of the progressive rise in non-communicable diseases. Thus, the continuous improvement in registries is required to improve capturing of ESKD patients for providing accurate data to health authorities, and enhancing public awareness of the magnitude, future trends, treatments, and outcomes regarding ESKD.

\subsection{Level of Satisfaction on Patient Care in Renal Dialysis Unit}

Several factors were identified as affecting hemodialysis patients' satisfaction with their dialysis therapy [13]. There were 322 recruited patients in the study where the male patients reported worse effect of dialysis on family life, social life, energy, and appetite. Longer period since the commencement of dialysis was associated with adverse effect on finances and energy. Lower level of education was associated with worse dialysis effect on stress, overall health, sexual life, hobbies, and exercise ability. The results imply that level of satisfaction is affected by gender, duration on dialysis, educational level, and standard of care given.

The concept of health-related quality of life $(\mathrm{HRQoL})$ for patients with end-stage renal disease on haemodialysis in the eastern region of Saudi Arabia [14] was carried out and seven themes defining HRQoL emerged from the qualitative data which reflected the subjectivity of the concept. The domains of HRQoL were the physiological, social, psychological, religious and vocational domains. Each of those domains was defined by determinants that affect other domains and the overall HRQoL. These were synthesised into a single definition, which incorporated all five domains. A model of HRQoL of patients undergoing haemodialysis in Saudi Arabia was developed explaining the relationship between the key domains and HRQoL. Healthcare services were discussed as a factor that affects the health status of patients and plays an indirect role in the life quality of this patient group. Hence it was considered as a factor rather than a domain. This illustrates that the concept of HRQoL is highly individual and is affected by the views of individuals living in a specific culture. Additionally, it is affected by the social, cultural and religious backgrounds of those individuals. The conceptualisation of HRQoL in this study was almost similar to the Western conceptualisation, however, the relationships between the key domains of HRQoL and their determinants differed. The social and religious domains were highly influenced by the Arabic culture and the Islamic religion. HRQoL in patients with ESRD in the Eastern Region of Saudi Arabia has a different meaning and conceptualisation from that seen in comparable Western literature. The assessment of HRQoL of patients with renal failure and on haemodialysis in Saudi Arabia requires a culturally-adapted instrument. This would ensure valid and reliable results that could be used in decision-making and the planning of care.

A cross-sectional study was used which focused on patients' satisfaction with care at dialysis unit and the study sample was selected from Beni-Suef University Hospital. This study involved 79 patients on hemodialysis. The sample has slightly more males (59.5\%) and the majority of the patients are married (74.7\%), and their education was mostly basic (read and write) $(48.1 \%)$ or primary $(26.6 \%)$. Whereas more than half of the patients on dialysis therapy between 1 and 5 years and Significant variation in all dimensions reported by patients were unsatisfied except time spent with doctor (64.6) and accessibility \& convenience (57.0). Moreover technical quality was good $(53.2 \%)$. Furthermore, the correlation between patients' satisfaction dimensions and patients personal characteristics' there were highly significant correlation $(\mathrm{P}=.003)$ between sex and technical quality, while marital status was negatively correlated $(\mathrm{P}=-.042)$ with time spent with doctor. Nonetheless, our results suggest that almost half of the study sample reported in general Satisfaction were unsatisfied, however the financial aspect, communication and interpersonal manner should be achieved to improve health care outcome then subsequently increase level of patients' satisfaction, and further research regarding patient satisfaction is required [15].

Another study on patients' satisfaction toward nursing care at hemodialysis units was conducted [16]. This descriptive study was carried out at hemodialysis units of Baghdad teaching hospitals from Feb. $4^{\text {th }}, 2010$ through Sep. $5^{\text {th }}, 2010$. A purposive (non-probability) sample of (150) patients at the hemodialysis units in Baghdad teaching hospitals was selected. The data were collected through the use of constructing questionnaire and by means of an interview technique with the patients; the questionnaire consists of two parts (1) demographic data (2) patients' satisfaction to nursing care. The validity of the study questionnaire was 
determined through the panel of experts and the reliability of the study questionnaire was determined through the pilot study. Descriptive statistical analysis procedure (frequency, percentage and mean of score) and inferential analysis procedure (person correlation coefficient, chi-square test and t-test) were used for data analysis. The findings of the study indicated that there is high satisfaction to clinical nursing care, communication/patient-nurse relationship and moderate satisfaction toward nursing health education. Not statistical significant difference between (gender, age, educational status, marital status, occupational status, frequency of hemodialysis and duration of hemodialysis) while there is a statistical significant difference between patients' occupational status and their satisfaction toward communication /patient-nurse relationship.

\subsection{Concerns of Patient Undergoing Hemodialysis}

One of the many concerns of patients undergoing hemodialysis in Saudi Arabia is insomnia. Studies have shown that insomnia is a common sleep disorder among patients with end-stage renal disease (ESRD). Using observational cross-sectional study which carried out over a period of five months in two hemodialysis centers in Saudi Arabia, the study found out that out of 227 enrolled patients, insomnia was reported by $60.8 \%$. The mean patient age was $55.7 \pm 17.2$ years; $53.7 \%$ were male and $46.3 \%$ were female. Insomnia was significantly associated with female gender, afternoon hemodialysis, Restless Legs Syndrome, high risk for obstructive Sleep Apnea Syndrome and excessive daytime sleepiness (P-values: 0.05, 0.01, < 0.0001, <0.0001, and $<0.0001$, respectively). No significant association was found between insomnia and other variables, including BMI, smoking habits, underlying etiology of renal failure, dialysis duration, association with hemoglobin, ferritin, and phosphorus or dialysis adequacy as measured by the Kt/V index. The results signify that indeed insomnia is common in dialysis patients and is significantly associated with other sleep disorders. Greater attention needs to be given to the care of dialysis patients with regard to the diagnosis and management of insomnia and associated sleep disorders [17].

\subsection{Health Care Providers' Experiences on Patient Care in Renal Dialysis Unit}

A quantitative exploration of the barriers and facilitators to nurse-patient communication was conducted in Saudi Arabia. This study aimed to investigate barriers and facilitators of nurse-patient communication in Saudi Arabia using the Nurses' Self-Administered Communication Survey. The survey was distributed to a random sample of 291 nurses working in medical and surgical departments at five hospitals in Saudi Arabia. The results indicate that the Filipino and Saudi Arabian nurses perceived greater barriers to communication with respect to personal/social characteristics, job specifications and environmental factors then nurses of other nationalities. In addition, nurses with shorter experience in Saudi Arabia perceived greater barriers to communication with respect to the clinical situation of patient and environmental factors than the nurses with longer experience. Lastly, nurses who had not attended specialist courses on communication skills acquisition perceived greater barriers to communication with respect to personal characteristics and job specifications than nurses who had attended such courses [18].

Furthermore, another study which evaluated nurses' practices throughout hemodialysis treatment for patients in hemodialysis unit at Baghdad teaching hospitals was carried out [19]. This descriptive study was conducted at hemodialysis units of Baghdad teaching hospitals started from December $25^{\text {th }}, 2011$ through May, $9^{\text {th }}, 2012$. Non-probability (purposive sample) of (30) nurses, who were working in hemodialysis units, were selected from Baghdad teaching hospitals. The data were collected through the use of constructed questionnaire, which consist of two parts (1) Demographic data form that consist 10-items and (2) Nurses' practices form that consist of 25 items, by means of direct interview technique and visualization of the nurses. The findings of the study indicated that there was a deficit in the nurses' practice that should be applied to the patient throughout hemodialysis treatment. No significant relationship was found between nurses' practice and their gender, level of education, years of experience in hemodialysis units, while significant relationship was found between nurses' practice and their marital status. The study recommended that the importance of employing Academic nurse in Hemodialysis units, a booklet should be designated and distributed to all nurses who were working in hemodialysis units including the standard actions that should be applied and followed to care for patients in hemodialysis units, and an educational program should be designated to the nurses who were working in hemodialysis units including the standard actions that should be applied to care for patients in hemodialysis units

\subsection{Barriers and Facilitators on Patient Care in Renal Dialysis Unit}

The knowledge, barriers and facilitators of exercise in dialysis patients was measured through a qualitative study among patients, staff and nephrologists. Despite the growing evidence on benefits of increased physical activity in hemodialysis (HD) patients and safety of intra-dialytic exercise, it is not part of standard clinical care, resulting in a missed opportunity to improve clinical outcomes in these patients [20]. In-depth interviews were conducted with a purposive sample of HD patients, staff and nephrologists from 4 dialysis units. Sixteen (16) in-center HD patients participated in the study (mean age 60 years, 50\% females, 63\% 
blacks), 14 dialysis staff members (6 nurses, 3 technicians, 2 dietitians, 1 social worker, 2 unit administrators) and 6 nephrologists (50\% females, $50 \%$ in private practice). Although majority of the participants viewed exercise as beneficial for overall health, most patients failed to recognize potential mental health benefits. Most commonly reported barriers to exercise were dialysis-related fatigue, co-morbid health conditions and lack of motivation. Specifically for intradialytic exercise, participants expressed concern over safety and type of exercise, impact on staff workload and resistance to changing dialysis routine. One of the most important motivators identified was support from friends, family and health care providers. Specific recommendations for an intra-dialytic exercise program included building a culture of exercise in the dialysis unit, and providing an individualized engaging program that incorporates education and incentives for exercising. This shows that patients, staff and nephrologists perceive a number of barriers to exercise, some of which may be modifiable. Participants desired an individualized intra-dialytic exercise program which incorporates education and motivation, and they provided a number of recommendations that should be considered when implementing such a program.

A study on patients' perception of comfort facilitators during hemodialysis procedure was conducted using qualitative method. Twenty four patients receiving hemodialysis participated in this study by purposive sampling. The sampling was over when the data saturation occurred. The semi-structured interviews were applied as the main data gathering tool. The data analysis was conducted by conventional qualitative content analysis in eight phases where three themes emerged in the study such as the presence of competent nurses, the delightful presence of the others, and coping with comfort obstacles. Each theme consisted of some categories. It seems that to achieve the patients' comfort during the hemodialysis procedure, the health care teams, hospitals in charge and the patients themselves have to do their best to provide the patients' comfort [21].

Patients undergoing hemodialysis require direct and continuous care. Identifying the barriers to and factors facilitating hemodialysis care can improve care quality. Another study was conducted which aimed to assess the barriers and facilitators of care for hemodialysis patients. It utilized qualitative study and content analysis approach. The study was performed in hemodialysis ward of Kowsar hospital in Semnan, in 2014. The main topic of health care challenges was divided into two main categories, including the facilitators and barriers of hemodialysis care. The facilitators of hemodialysis care had four subcategories, including intimate relationship, basic knowledge, hemodialysis skills, and experience. The category of barriers had eight subcategories, including shortage of nurses and heavy workload, weak authority of the head nurse, ignorant director of nursing, shortage of nephrologists, lack of vascular surgery expert, lack of nurse's aide and nursing assistant, unskilled staffs, and interference by patients' caregivers. The findings of this study showed that access to human resources and their abilities were among the factors facilitating care. However, lack of qualified medical staff at each level of care delivery was one of the barriers to hemodialysis care. Hence, it is of great importance for policy makers, managers, and program designers to recruit human resources who have the characteristics and competencies required for providing hemodialysis care. In a study on a group of hemodialysis patients, in Semnan of Iran, it was found that access to human resources and their abilities were among the factors facilitating care. However, lack of qualified medical staff at each level of care delivery was one of the barriers to hemodialysis care. Hence, it is of great importance for policy makers, managers, and program designers to recruit human resources who have the characteristics and competencies required for providing hemodialysis care [22].

\section{Discussion}

The burden of Chronic Kidney Disease (CKD) is increasing in alarming proportion all over the world. Kidneys are probably the only vital organs which can be realistically replaced by artificial means. Maintenance dialysis is a well-recognized modality of treating patients having end stage renal disease. Several thousands of patients all over the world are surviving and achieving reasonable quality of life on maintenance dialysis.

Hemodialysis therapy is a very complex and challenging area of patient with end stage renal disease [23-24]. However, assessment of patients' satisfaction is very important to evaluate the health care outcomes and these are becoming increasingly important in health care delivery [25-26]. There is evidence that better patient satisfaction might be associated with better medical outcome including reduced hospitalization [27] and reduced mortality [28].

Patient satisfaction is very important aspect to evaluate health care outcome, so patient satisfaction has been often defined as the extent of agreement between what a patient expects to result or obtain from the healthcare experience and the perception of care they actually receive [29]. It is considering as an important indicator for evaluating the health care outcomes, while affects clinical performance, patient retention and medical malpractice. Furthermore it may be a very effective indicator to measure the success of health care team especially in dialysis unit when deal with chronic patient undergoing hemodialysis therapy [30].

Chronic kidney disease is a major health problem due to the significant financial burden for the healthcare system and likewise for the patient who needs the treatment. The patient's whole life situation is 
turned upside down with chronic kidney disease when they are confronted with the forced change to start treatment with hemodialysis.

The importance of the nurses' and doctors' sincere and genuine interest in the patient as an individual provides support to the patient [31]. The nurse affects the quality of the support when she shows an interest in the patients through exhibiting openness during the meeting, which creates opportunities for them to participate as well as for her to familiarize herself with the patients' entire lives. The intimate relationships are powerful indicators of support for patients [32].

The delivery of continuous and coordinated care lessens the demand for hospital services, waiting times for other services in different parts of the hospital, and the threat of complications [33-34]. That is why patients treated with hemodialysis not only have a low health related quality of life as [35] as well as eexperienced mortality rates of $15 \%-20 \%$ each year [36].

The patients and their family members are always in need of care, guidance, training, and ongoing support provided by the health team from the hospital. Support in caring for patients with chronic renal failure (chronic kidney disease) poses a challenge for nurses and the entire health team. Assistance should be designed with the intention that the patient must maintain as high a quality of everyday life as possible, while taking into account the medical data of the patient [37].

Culture plays a vital role in the patients' eating and lifestyle behaviors which could affect the management of kidney disease. Relationships and social activities will likewise challenge the nutritional management and fluid control for HD patients [38]. The main barriers to the delivery of care in hemodialysis patients include the following: forgetfulness, safety concerns, lack of understanding and knowledge, poor communication, and lack of control over social pressures [39]. However, the factors facilitating hemodialysis care include internal factors (self-starting factors) and external factors (started by parents, professionals, other professional members of the health care team, and other hemodialysis patients).

On the other hand, the explanations presented by care team members and also the relationship with other dialysis patients are among the facilitators of dialysis care [40]. The support amidst the struggles of HD patients and their families is very crucial. Because of various diseases and physical and psychological problems, this group of patients is in need of constant and comprehensive patient care to ensure their adherence to therapy. However this type of care is seldom provided for patients. In many hospitals and/or dialysis centers, the delivery of health care services by health care providers is very complex and people who are affected by the problems have different levels of sensitivity to the treatment. However, the existing evidences suggest that using a promising approach, these conditions can be appropriately managed and the outcomes of intervention could be improved for this vulnerable population [41].

This indicates that the health provider team, hospital officials and the patient himself should do their utmost to let hemodialysis patients achieve comfort, which is not possible without the intervention of nurses, doctors, and hospital officials. Nurses and doctors play a significant role in comforting these patients because they have the most direct contact with them and are able to identify and provide comforting elements. Nurses and doctors play a key role in the treatment team. They can form suitable relationship, as sist to create positive attitude in the patient, and provide an appropriate caring context for them. Besides, to a large extent officials can help patients achieve their aims by providing welfare, cultural and ritual facilities, and allowing the presence of family members and visitors along with observing the others' rights and security issues.

This study highlights the need to better prepare expatriate nurses before they enter the workforce in Saudi Arabia on cultural competence and language skills.

\section{Conclusion}

This review presents an overview of the increasing burden of ESRD in the GCC countries, which is similar to the rest of the world. It also provides a list of the major causes of ESRD in these countries and the most frequent morbidities associated with ESRD.

The available data also do not provide an accurate and updated estimate for relevant outcomes. Additionally, considering the increasing burden of chronic kidney disease (CKD), these results stressed the needs and the importance of preventative strategies for leading causes of ESRD. Furthermore, more studies are needed to describe the epidemiology of ESRD and for assessing the overall quality of renal care.

Many barriers continue to restrict access to maintenance dialysis among the ESRD population. As efforts are being made to tackle these issues, other challenges are assuming greater prominence. The findings of this study showed that access to human resources and their abilities were among the factors facilitating care; however, lack of qualified medical staff at each level of care delivery was one of the barriers to hemodialysis care. Hence, it is of great importance for policy makers, managers, and program designers to recruit human resources who have the characteristics and competencies required for providing hemodialysis care. The facilitators of hemodialysis care had four subcategories namely intimate relationship, basic knowledge, hemodialysis skills, and experience. The category of barriers includes shortage of nurses and heavy workload, 
weak authority of the head nurse, ignorant director of nursing, shortage of nephrologists, lack of vascular surgery expert, lack of nurse's aide and nursing assistant, unskilled staffs, and interference by patients' caregivers. The findings of this study showed that access to human resources and their abilities were among the factors facilitating care. However, lack of qualified medical staff at each level of care delivery was one of the barriers to hemodialysis care.

\section{Acknowledgements}

This work is part of the researcher's Doctoral study undertaken through the Universiti Malaysia Sarawak (UNIMAS). A note of appreciation goes to the researcher's Adviser Associate Professor Dr. Zabidah Binti Putit, Deputy Dean, Faculty of Medicine and Health Sciences and co-adviser Dr. Sidiah John Siop, Department of Nursing, Faculty of Medicine and Health Sciences, Universiti Malaysia Sarawak (UNIMAS)

\section{References}

[1]. J. M. Bargman and K. Skorecki, Chronic kidney disease in Fauci A. 208, (17 ${ }^{\text {th }}$ Edition New York: McGraw Hill, 2008) 2308-2321

[2]. R.K. Agrawal, S. Khakurel, R. Hada, D. Shrestha, and A. Baral, Acute intradialytic complications in end stage renal disease on maintenance hemodialysis, JNMA J Nepal Med Association, 52(187), 2012, 118-21.

[3]. M.R. Tamadon, et al., Evaluation of noninvasive tests for diagnosis of Helicobacter pylori infection in hemodialysis patients, $J$ Nephropathol, 2(4), 2003, 249-53.

[4]. R.J. Glassock, The rising tide of end-stage renal disease: what can be done? Clin Exp Nephrol, 8 (4), $2004,291-296$.

[5]. A.N. Haghighi, et al., The epidemiology of end-stage renal disease in Iran in an international perspective, Nephrol Dial Transplant, $17(1), 2002,28-32$.

[6]. A.J. Collins, et al., United States Renal Data System 2011 Annual Data Report: Atlas of chronic kidney disease \& end-stage renal disease in the United States, Am J Kidney Dis, 59(1 Suppl 1), 2012, eA7 e1-420.

[7]. US Renal Data System, USRDS, Annual Data Report: Atlas of Chronic Kidney Disease and End-Stage Renal Disease in the United States. Bethesda: National Institutes of Health, National Institute of Diabetes and Digestive and Kidney Diseases, 2013. Available from: http://www.usrds.org/atlas13.aspx. Accessed April 5, 2017

[8]. A. Rastogi, A. Linden, and A.R. Nissenson, Disease management in chronic kidney disease, Adv Chronic Kidney Dis, 15(1), 2008, 19-28.

[9]. A. A. Hassanien, et al., Epidemiology of end-stage renal disease in the countries of the Gulf Cooperation Council: a systematic review, Journal of the Royal Society of Medicine Short Reports, 3(6), 2012, 1-22

[10]. H. Al-Marhuby, Renal Replacement Therapy in Sultanate of Oman, Saudi J Kidney Dis Transplant, 9(4), 1999, 459-460

[11]. O.M. Fituri, M.M. Shigidi, G. Ramachandiran, A.H.Rashed, Demographic data and hemodialysis population dynamics in Qatar: A five year survey. Saudi J Kidney Dis Transpl, 20(3), 2009, 493-500

[12]. Al Ismaili, Faisal et al., Epidemiological Transition of End-Stage Kidney Disease in Oman, Kidney International Reports, 2(1), $2017,27-35$

[13]. Al Eissa, M. Al Sulaiman, M. Jondeby, et al., Factors Affecting Hemodialysis Patients' Satisfaction with Their Dialysis Therapy, International Journal of Nephrology, Vol. 2010, Article ID 342901, 5 pages, 2010. doi:10.4061/2010/342901

[14]. R. S. Algarni, Exploring the Concept of Health-related Quality of Life for Patients with End-stage Renal Disease on Haemodialysis in the Eastern Region of Saudi Arabia, doctoral dissertation, School of Nursing Midwifery and Social Work, University of Manchester, 2014

[15]. M. Bayoumi, A. Hoda, and A. Ahmed, Patients' Satisfaction with Care at Dialysis Unit, International Journal of Nursing Science, 6(5), 2016, 117-122

[16]. A. T. Shnishil and K. A. Mansour, Assessment of Patients' Satisfaction toward Nursing Care at Hemodialysis units, Iraqi National Journal of Nursing Specialties, 26(1), 2013, 1-9

[17]. H. Al-Jahdali et al., Insomnia in chronic renal patients on dialysis in Saudi Arabia, Journal of Circadian Rhythms, 8(7) 2010, 1-7

[18]. B. Albagawi and L. K. Jones, Quantitative exploration of the barriers and facilitators to nurse-patient communication in Saudi Arabia, Journal of Hospital Administration, 6(1), 2017

[19]. S. J. Bakey, Evaluation of Nurses' Practices throughout Hemodialysis Treatment for Patients in hemodialysis unit at Baghdad teaching hospitals, KUFA Journal for Nursing Science, 2(2), 2012, 1-16

[20]. Jhamb, M., McNulty, M.L., Ingalsbe, G. et al. Knowledge, barriers and facilitators of exercise in dialysis patients: a qualitative study of patients, staff and nephrologists, BMC Nephrol, 17(192), 2006.

[21]. S. R. Borzou, M. Anosheh, E. Mohammadi, and A. Kazemnejad, Patients' Perception of Comfort Facilitators During Hemodialysis Procedure: A Qualitative Study, Iran Red Crescent Med J, 16(7), 2014, e19055.

[22]. M. Nobahar and M.R.Tamadon, Barriers to and facilitators of care for hemodialysis patients; a qualitative study. $J$ Renal Inj Prev. 5(1), 2016, 39-44.

[23]. M.A. Roberts, K.R. Polkinghorne, S.P. McDonald, and F.L. Lerino, Secular trends in cardiovascular mortality rates of patients receiving dialysis compared with the general population, Am J Kidney Dis, 58(1), 2011, 64-72

[24]. H. B. Neuman, J. Park, M.R. Weiser, Randomized clinical trials in colon cancer, Surg Oncol Clin N Am, 19(1), 2010, $183-204$.

[25]. M.L. Unruh, S.D. Weisbord, and P.L. Kimmel, Psychosocial Factors in Patients With Chronic Kidney Disease: Health-Related Quality of Life in Nephrology Research and Clinical Practice, Seminars in Dialysis, 18, 2005, 82-90. doi:10.1111/j.1525139X.2005.18206.X

[26]. J. Cancato and A.R. Feinstein, Asking Patients What They Like: Overlooked Attributes of Patient Satisfaction with Primary Care, Am J Med, 102(4), 1997, 399-406

[27]. P.L. Kimmel and R.A. Peterson, Depression In End-Stage Renal Disease Patients Treated With Hemodialysis: Tools, Correlates, Outcomes, And Needs, Seminars in Dialysis, 18 (2), 2005, 91-97

[28]. D. L. Mapes, et al., Health-related quality of life as a predictor of mortality and hospitalization: the Dialysis Outcomes and Practice Patterns Study (DOPPS). Kidney Int. 64(1), 2003, 339-49.

[29]. E. L. LaMonica, M.T. Oberst, A.R. Madea, and R.M. Wolf, Development of a Patient Satisfaction Scale, Research in Nursing and Health, 9(1), 1986, 45-50.

[30]. B. Prakash, Patient satisfaction, J Cutan Aesthet Surg. 3(3), 2010, 151-5. 
[31]. K. Ziegert, B. Fridlund, and E. Lidell, Professional support for next of kin of patients receiving chronic haemodialysis treatment: A content analysis study of nursing documentation, Journal of Clinical Nursing, 16, 2007, 353-361.

[32]. M. Stewart, K. Davison, D. Meade, and A. Hirth, Weld-Viscount P. Group support for couples coping with a cardiac condition, Journal of Advanced Nursing, 33(2), 2001, 190-199.

[33]. L.M. Tejada-Tayabas, K.L. Partida-Ponce, L.E. Hernandez-Ibarra, Coordinated hospital-home care for kidney patients on hemodialysis from the perspective of nursing personnel, Rev Lat Am Enfermagem, 24(1), 2015, 225-33.

[34]. M. Nobahar and M.R. Tamadon, Barriers to and facilitators of care for hemodialysis patients; a qualitative study, J Renal Inj Prev, 5(1), 2016, 39-44. DOI: 10.15171/jrip.2016.09

[35]. M. Wyld, R. L. Morton, A. Hayen, K. Howard, and A.C. Webster, A systematic review and meta-analysis of utility-based quality of life in chronic kidney disease treatments, PLoS Med, 9(9), 2012, e1001307

[36]. US Renal Data System, USRDS, Annual Data Report: Atlas of Chronic Kidney Disease and End-Stage Renal Disease in the United States. Bethesda: National Institutes of Health, National Institute of Diabetes and Digestive and Kidney Diseases, 2013. Available from: http://www.usrds.org/atlas13.aspx. Accessed April 5, 2017

[37]. S.E. Thorne, S.R. Harris, K. Mahoney, A. Con, \& L. McGuinness, The context of health care communication in chronic illness, Patient Education and Counseling, 54(3), 2004, 299-306.

[38]. A. Warde \& L. Martens, Social differentiation, consumption and pleasure. (Cambridge: Cambridge University Press, 2000)

[39]. K. Griva, H.J. Ng, J. Loei, N. Mooppil, H. McBain, and S. P. Newman, (2013). Managing treatment for end-stage renal disease- - a qualitative study exploring cultural perspectives on facilitators and barriers to treatment adherence, Psychol Health. 28(1), 2013, 1329.

[40]. K. B. Chenitz, M. Fernando, and J. A. Shea, In-center hemodialysis attendance: patient perceptions of risks, barriers, and recommendations, Hemodial Int, 18(2), 2014, 364-73.

[41]. A. Rastogi, A. Linden, and A.R. Nissenson, Disease management in chronic kidney disease, Adv Chronic Kidney Dis, 15(1), 2008, 19-28. 